\title{
Distinguirse en Internet: la desambiguación de la firma
}

\section{Distinguish yourself on the Internet: signature disambiguation}

\author{
Sara MANDIÁ-RUBAL \\ Universidade de Santiago de Compostela, USC (España) \\ sara.mandia.rubal@gmail.com \\ https://orcid.org/0000-0003-4452-2751 \\ Maricela LÓPEZ-ORNELAS \\ Universidad Autónoma de Baja California, UABC (México) \\ ornelas@uabc.edu.mx \\ https://orcid.org/0000-0002-4215-5591 \\ Recepción: 4/12/2020 Revisión: 10/12/2020 \\ Aceptación: 20/12/2020 Publicación: 27/12/2020
}

\section{Resumen}

Los autores no son conscientes de la cantidad de personas que publican cada año, ni de la facilidad para encontrar idénticos nombres y apellidos en el conjunto de un país. Un problema que, con Internet, y la muerte de la distancia en la diseminación de información, se agrava aún más. Hace años que en comunicación científica se trata de ofrecer herramientas útiles en la desambiguación de autoridades. Conviven, sistemas de identificación de autor por codificación numérica o alfanumérica; sistemas de perfil personal-profesional puros; recursos híbridos; y, catálogos de autoridades, la herramienta clásica de normalización en bibliotecas. Además de un análisis descriptivo de los citados sistemas, se facilitan, a modo de cierre, algunas indicaciones para los escritores noveles, y no tan noveles, a la hora de diferenciarse en el magma de autores existente. Datos tan simples como la inserción de un segundo apellido unido con guion, facilitar el año y lugar de nacimiento, la profesión, mención a otros trabajos, o el enlace a un sistema de identificación como los señalados, son posibilidades que, añadidas a los materiales a editar, aportan ese punto distintivo y contribuyen a crear una marca personal por la que ser reconocidos y reconocibles.

\begin{abstract}
With the Internet, the problem of correctly and unambiguously identifying the author of a document has been further aggravated by the amount of information that is housed and the lack of a controlled language that avoids homonyms and noise in the retrieval of said information. At present, author identification systems by numerical or alphanumeric coding coexist; personal-professional profile systems; hybrid or combined resources; and, the authority catalogs, the classic identification and standardization tool in library science. In addition to a descriptive analysis of identification systems, some pointers are provided for novice and not-sonovice writers on avoiding ambiguities. Data as simple as inserting a second surname, providing the year and place of birth, profession, mention of other jobs, if any, or the link to an identification system such as those mentioned above, are possibilities that, added to Materials to be edited, provide that distinctive point and contribute to creating a personal brand by which to be recognized and recognizable.
\end{abstract}

Palabras Clave: autoridad; catálogo de autoridades; España; Internet; perfil profesional; sistemas de identificación.

Keywords: authorities' catalogue; authority; Internet; personal digital identifier; professional profile; Spain. 


\section{Introducción}

La llegada y posicionamiento de las tecnologías de la información y la comunicación en el contexto científico de la información, han generado un universo de transformaciones - Internet se visualiza como el más contundente-. Este tipo de metamorfosis ha exigido ajustes para clasificar, recuperar, acceder y visibilizar la información, por tanto, en las últimas décadas - a nivel internacional一, se ha producido la necesidad de normalizar su integración en los sistemas de información, y las bibliotecas no son la excepción; bajo esta perspectiva, entre los aspectos más significativos de este proceso tecnológico, se encuentra la normalización catalográfica (Picco y Ortiz-Repiso, 2012).

Al respecto, el Ministerio de Educación y Cultura (1999), expone qué:

La normalización catalográfica es un elemento vertebral en la recuperación adecuada de la información en tratamientos manuales que resulta imprescindible cuando se utilizan las tecnologías de información y comunicación. Con esta publicación la Dirección General del Libro, Archivos y Bibliotecas pretende contribuir a facilitar en última instancia, el intercambio y el aprovechamiento de la información bibliográfica entre bibliotecas españolas de forma normalizada y el acceso a la misma por parte de los ciudadanos (p. 7).

Al respecto, la Biblioteca Nacional de España ([BNE], 2018), define el catálogo de autoridades como "el conjunto de registros de autoridad de nombres de personas, entidad corporativa, título y materias" (párr. 1), conjuntamente añade, que dicho catálogo refleja la investigación realizada por la Biblioteca Nacional, que contiene, además de la forma aceptada para cada autoridad.

Por tanto, cabe reconocer, que Internet si bien ha abierto un mundo de posibilidades para acceder a la información, también ha generado un alto nivel de infoxicación (Portugal y Aguaded, 2020), generando la invisibilidad sin la distinción un perfil digital o el establecimiento de sistemas de información que especifiquen, validen y consoliden la autoría de lo difundido en la red.

Por ende, la necesidad de distinguirse académicamente, no es un tema nuevo en la normalización de la firma dentro del ámbito científico. Según se ha citado, resulta paradigmático el rol que actualmente tienen los generadores de conocimiento en la autoedición de sus diversos perfiles digitales (Barroso-Osuna, et al., 2020) —servicio cada vez más demandado, y en el que se vuelven parcialmente responsables sobre la visibilidad de su producción científica-.

En consonancia, la duplicidad de nombres y apellidos en el contexto académico que se divulgan en los diversos géneros de la comunicación científica formal e informal, se ha transformado en un 
problema complejo dentro de la comunidad académica a nivel internacional (Díaz-Redondo y FríasMontoya, 2018).

La identidad digital es el resultado del esfuerzo consciente que realiza el investigador por y para ser identificado y reconocido en un contexto digital, distinguiéndose del conjunto de investigadores a través de la normalización, con el uso de identificadores, y la difusión de resultados de investigación en redes y plataformas de diversa naturaleza (p. 12).

Como ejemplo, la siguiente Tabla 1 muestra la cantidad de personas con un mismo apellido, en primera y segunda posición; no digamos la combinación de dos distintos apellidos, advertidos de la misma lista. Si bien esta información se refiere a España, es evidentemente claro, que esta problemática que no concierne exclusivamente a un país.

Tabla 1: INEbase. Frecuencias de apellidos en España

Apellido $1^{\circ}$

Total Por mil (\%)

\section{Apellido $2^{\circ}$}

Total Por mil (\%)

Ambos apellidos

$\begin{array}{lrrrrr} & \text { Total } & \text { Por mil (\%) } & \text { Total } & \text { Por mil (\%) } & \text { Total } \\ \text { García } & 1.462 .923 & 31,109 & 1.480 .844 & 31,49 & 78.393 \\ \text { Rodríguez } & 927.056 & 19,714 & 936.885 & 19,923 & 42.447 \\ \text { González } & 925.695 & 19,685 & 934.490 & 19,872 & 40.843 \\ \text { Fernández } & 912.009 & 19,394 & 922.708 & 19,621 & 50.777 \\ \text { López } & 869.944 & 18,499 & 877.771 & 18,666 & 34.073 \\ \text { Martínez } & 832.525 & 17,703 & 838.318 & 17,827 & 38.330 \\ \text { Sánchez } & 816.968 & 17,373 & 826.050 & 17,566 & 33.968 \\ \text { Pérez } & 777.950 & 16,543 & 791.231 & 16,825 & 25.891 \\ \text { Gómez } & 491.596 & 10,454 & 495.549 & 10,538 & 13.646 \\ \text { Martín } & 486.852 & 10,353 & 485.063 & 10,315 & 20.862\end{array}$

Fuente: Elaboración y selección propia.

Quizá en principio, la elección de la firma podría parecer una cuestión superficial, pero no lo es, y al mismo tiempo, debe realizarse sin perder de vista el objetivo de del proceso, esto es, la generación de una identidad bibliográfica única que consolide la producción del investigador de manera unívoca y bajo un solo encabezamiento, que requiere ser el nombre exacto o completo del 
autor (Díaz-Redondo y Frías, 2018). Pues como lo expresan Barroso-Osuna et al, (2020) en Sampedro Requena (2015) "Uno de los aspectos más preocupantes a tener en cuenta para los estudiantes de Educación en la actualidad, es tener presente el concepto sobre la identidad digital" (p. 55).

La Figura 1, ejemplifican con 30 entradas, el problema de la desambiguación de la firma de autores, ya que de acuerdo a los datos proporcionados en dicha figura, incluso incluyendo los dos apellidos del autor —en este caso la búsqueda utilizó los datos de "Fernández Hernández" - , se observa, cómo la Biblioteca Nacional de España añade en algunos autores, datos como fecha de nacimiento, nacimiento y fallecimiento, e información, para identificar sus obras.

Figura 1: Treinta primeras entradas para la búsqueda "Fernández Hernández"

\begin{tabular}{ll} 
Fernández Hernández, Aida & Obras \\
Fernández Hernández, Amparo & Obras \\
Fernández Hernández, Antonio & Obras \\
Fernandez Hernández, Bernabé & Obras \\
Fernández Hernández, Caridad & Obras \\
Fernández Hernández, Caridad Ruta literaria de Carmen & Obras \\
Conde & \\
Fernández Hernández, Carlos 1963- & Obras \\
Fernández Hernández, Carmen María & Obras \\
Fernández Hernández de Castilleja, Pedro ca. 1487-1574 & Obras \\
Fernández Hernández, Delia & Obras \\
Fernández Hernández, Emiliana & Obras \\
Fernández Hernández, Felipe & Obras \\
Fernández Hernández, Francisco J. & Obras \\
Fernández Hernández, Gonzalo & Obras \\
Fernández Hernández, Gonzalo Teoría...grado superior de & Obras \\
informática & Obras \\
Fernández Hernández, Irene & Obras \\
Fernández Hernández, Isidoro & Obras \\
Fernández Hernández, Iván & Obras \\
Fernández Hernández, J. & Obras \\
Fernández Hernández, J. A. & Obras \\
Fernández Hernández, Javier & Obras \\
Fernández Hernández, Jesús & Obras \\
Fernández Hernández, José & Obras \\
Fernánández & Obras \\
Fernández & Obras \\
\hline & Obras \\
\hline
\end{tabular}

Fuente: Catálogo de autoridades de Biblioteca Nacional de España. 
Por tanto, resulta sumamente relevante que todos los relacionados con la producción, publicación, gestión, almacenamiento y visibilidad de la ciencia, conozcan que "la competencia digital, demanda el desarrollo de conocimientos, habilidades y actitudes que permitan efectuar con pertinencia la gestión de la información con transparencia" (Portugal y Aguaded, 2020, p. 28). Esto no es un tema aislado, la carencia de este tipo de conocimientos, eleva la ambigüedad en la literatura científica especializada a nivel internacional, ya que de acuerdo con Barroso-Osuna et al, (2020)

La digitalización de la sociedad confiere a cada persona una identidad digital que crece y se desarrolla paralela a la identidad del mundo físico o real, generándose procesos relativos al manejo de información personal cada vez más intensivos, exigentes y automatizados que controlan nuestras vidas (p. 55).

Entre los distintos problemas de la duplicidad, puede ser, que los/las autores/as cuenten con una vasta trayectoria, y alguien con el mismo nombre y apellido firme igual, situación que no solo confundiría el perfil de la persona, sino además a lectores principiantes en la validación de datos de los autores consultados, por tanto, es necesario evitar la duplicidad de perfiles, y normalizar los nombres simples, compuestos o diminutivos (Siso-Calvo y Arquero-Avilés, 2020).

En el caso de las Figuras 1 y 2, se presentan dos de las múltiples eventualidades que acontecen regularmente en la recuperación de información a través de diversas bases de datos. Como ejemplo, se presenta el caso de las autoras que firman como "Ana María López" (véase Figura 1 y 2), y "Carmen Muñoz" (véase Figura 3 y 4).

\section{Figura 2: Ejemplo de firma ambigua en edición científica}

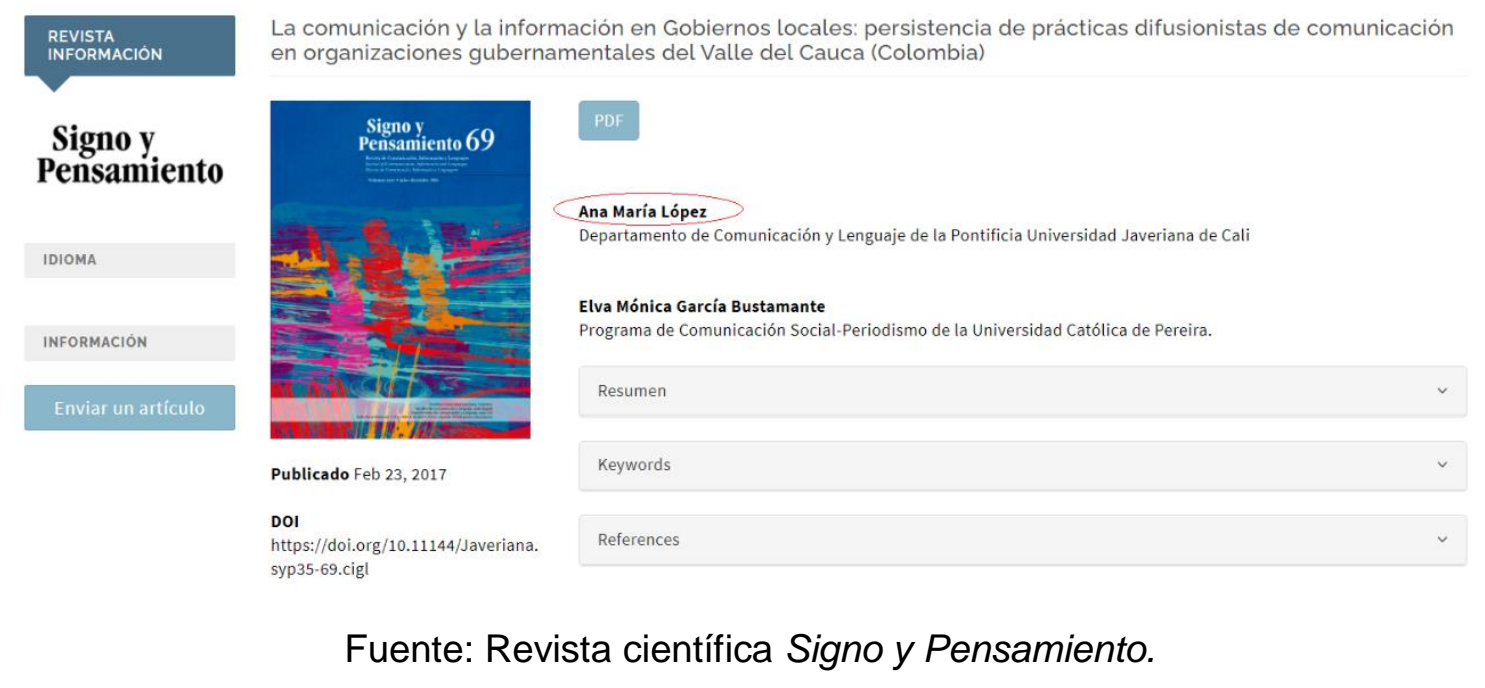

Figura 3: Ejemplo de firma ambigua en edición científica

Pangea. Revista de la Red Académica Iberoamericana de Comunicación ISSN: $2172-3168$ | https://revistapangea.org Vol.11, núm. 1 (2020) | Páginas: 42 -58- | DOI: xxxxxx 


\begin{tabular}{|c|c|c|c|}
\hline ORCID ID & First Name & Last Name Other Names & Affiliations \\
\hline 0000-0002-4088-7991 & Ana Maria & Lopez & \\
\hline 0000-0002-4898-9853 & Ana Maria & Lopez & \\
\hline 0000-0002-2759-5353 & Ana Maria & Lopez & $\begin{array}{l}\text { Bryn Mawr College (Haverford College), Huntsman Cancer Hospital, Sidney Kimmel } \\
\text { Cancer Center, Sidney Kimmel Medical College at Thomas Jefferson University, University } \\
\text { of Arizona, University of Arizona College of Medicine, University of Arizona College of } \\
\text { Public Health, University of Arizona, College of Medicine, University of Utah }\end{array}$ \\
\hline 0000-0003-0219-522X & Ana Maria & Lopez & Hospital Clinic de Barcelona, Universitat de Barcelona Facultat de Medicina \\
\hline 0000-0002-6569-5021 & Ana Maria & Lopez & \\
\hline $0000-0002-4857-4893$ & Ana María & López & $\begin{array}{l}\text { Tictag Branding Digital, Universidad de Murcia, Universidad de Murcia - Campus de } \\
\text { Espinardo }\end{array}$ \\
\hline
\end{tabular}

Fuente: Identificador Abierto de Investigador y Colaborador (ORCID, por su inglés).

Figura 3: Ejemplo de firma ambigua en edición científica

$$
\text { OXFORD }
$$

\section{APPLIED} LINGUISTICS

Issues Advance articles

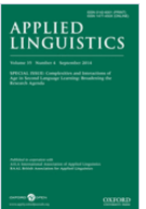

Volume 35, Issue 4 September 2014

Contrasting Effects of Starting Age and Input on the Oral Performance of Foreign Language Learners

\section{Carmen Muñoz $\mathrm{M}$}

Applied Linguistics, Volume 35, Issue 4, September 2014, Pages 463-482, https://doi.org/10.1093/applin/amu024 Published: 17 June 2014

$\mathbf{6 6}$ Cite 2 Permissions 2 Share $\mathbf{v}$

Fuente: Revista científica Applied Linguistics.

\begin{tabular}{lllll} 
ORCID ID & First Name Last Name & Other Names & Affiliations \\
\hline $0000-0002-4887-5258$ & Carmen & Muñoz & & \\
\hline $0000-0003-1323-3957$ & Carmen & Muñoz & & \\
\hline $0000-0003-4904-1957$ & carmen & munoz & & \\
\hline $0000-0002-7001-4155$ & Carmen & Muñoz & $\begin{array}{l}\text { Carmen Muñoz } \\
\text { Lahoz }\end{array}$ & University of Barcelona, University of Reading
\end{tabular}

Fuente: Identificador Abierto de Investigador y Colaborador (ORCID, por su inglés). 


\section{Bosquejo metodológico}

De una investigación en curso, estudiando una muestra de más de dos mil doscientos autores españoles o con filiación/adscripción en una universidad española entre los años 2011 y 2016 , cuyos artículos se hubieran publicado en revistas indexadas en SCImago Journal \& Country Rank, se logró constatar, que un 5,2\% de la muestra — de 125 autores-, requirió ser descartada por la ambigüedad de su firma, y la imposibilidad de clarificar que, efectivamente, los perfiles abiertos en la red, se correspondían siempre a la misma autoridad.

Al respecto, los 125 nombres descartados concernieron a autores de artículos publicados entre 2011 y 2016. Con todo, la tarea de normalización no finaliza necesariamente en el autor. Los directores de las revistas científicas pueden y deben participar en el proceso atendiendo a esta problemática en su política editorial y dictando una serie de recomendaciones dirigidas a los académicos en las normas de publicación de sus revistas. Entre sus responsabilidades, se podría recalcar, la de concienciar al autor de la trascendencia del uso de un nombre científico normalizado, requiriendo en los textos originales una firma con un identificador digital único, y facilitándole la información y herramientas que pudieran ser necesarias para crearlo (Díaz-Redondo y Frías, 2018).

Se reconoce igualmente, la existencia de literatura acerca de esta problemática — duplicidad—. Hasta el momento, si bien había sido algo opcional por parte de los autores, cada vez más, las revistas indizadas en importantes bases de datos, exigen a sus colaboradores, contar con un Identificador Abierto de Investigador y Colaborador (ORCID, por su inglés), incluso, como requisito para someter y publicar artículos (Poveda, Rasero, Fernández del Pino, \& Martín, 2017).

Este proceso de la legitimación de la identidad digital de los autores, no es del todo nuevo, ya que un par de años, que en la comunicación científica trata de ofrecer herramientas útiles en la desambiguación de autoridades, donde conviven sistemas de identificación de autor por codificación numérica o alfanumérica como el identificador de objeto digital (DOI, por sus siglas en inglés); sistemas de perfil personal-profesional puros como ORCID; recursos híbridos; y, catálogos de autoridades, la herramienta clásica de identificación y normalización en bibliotecología, de uso abierto y gratuitas para autores e interesados.

\section{Una aproximación al estado del arte}

La edición de libros en España, en datos del Ministerio de Cultura y Deporte (2018), asciende en 2017, a 89.962 libros. Un 4,6\% más que el año anterior. La edición de libros en soporte papel representa el $68,38 \%$ del total, con 61.519 libros; la edición en otros soportes copa el 31,62\%, con 
28.443 libros. Esta última, la edición en otros soportes, supone un incremento, con respecto al año 2016, del $12,7 \%$.

Los eBook o libros digitales aumentan el 16,4\% y representan el 96,8\% de la edición en otros soportes, y el 30,6\% del total de la producción. De los 89.962 libros editados en todo el año: 88.119 son primeras ediciones, el 97,95\%; y 1.843 reediciones, apenas el 2,05\%.

De acuerdo con el tipo de edición, el total de libros con Número Estándar Internacional de Libros (ISBN, por sus siglas en inglés) inscritos, observa una disminución en la edición de carácter público, de un $6,4 \%$ respecto a 2016; mientras que la edición privada aumenta un 5,8\%; representando en 2017 , el $8,6 \%$ y el $91,4 \%$ respectivamente.

En lo que a la edición de carácter privado respecta, y a la naturaleza jurídica del agente editor, cabe diferenciar, al Autor-Editor, con 5.094 libros editados en 2017, el 6,2\% del total, una cifra que año a año se incrementa en número notablemente; la pequeña editorial, encargada de publicar el 34,41\% del total, lo que hace que con 28.327 libros a su cargo, sea la parte más productiva en este año 2017; las editoriales medianas, que ha significado el 31,1\% del total, con 25.556 libros editados; y las grandes editoriales, que lejos de lo que pudiera parecer, han sido productoras materiales de 23.261 obras, apenas el $28,29 \%$ del total.

Las comunidades autónomas en España de Cataluña y Madrid, representan el 63,5\% del total de la producción, con una participación del 34,2\% para Cataluña, y del 29,3\% para Madrid; seguidas de Andalucía, 15,3\%; y la Comunidad Valenciana, con el 6,6\%.

Por subsectores de edición, en este 2017 se observan aumentos en el libro infantil y juvenil, con 11.269 libros y el $12,53 \%$ del total - un aumento con respecto al año 2016 , nada menos que del $21,0 \%$-; creación literaria, con 21.745 libros y el $24,17 \%$ del total —en este caso aumentando con respecto al año anterior, un 18,0\%—; libros de Ciencia y Tecnología, con 12.726 y el 14,15\% del total, observa un incremento del 5,1\%; libros de Ciencias Sociales y Humanidades, con 27.661 y la friolera del $30,74 \%$ del total de títulos editados, incrementándose en este caso el 3,8\% — parece que también en 2016, las Ciencias Sociales y las Humanidades, eran temas atractivos para la publicación de obras-; y libros de tiempo libre, que significando apenas el 7,11\%, con 6.395 libros, se incrementa en un 3,4\%. Los descensos se registran en los libros de texto, con un declive en relación al año anterior del 27,6\%; y en "otros", observando una bajada del 5,8\% respecto a los datos de 2016.

El 94,2\% de los libros se editaron en lenguas españolas: Castellano, 71.530 libros, el 79,51\% del total; catalán, 9.702 libros, el 10,78\% del total; euskera, 1.430 libros, y el 1,59\% del total; gallego, 
1.288 libros, y el 1,43\% del total; valenciano, 723 libros y el 0,80\% del total; otras lenguas españolas, 63 libros y el 0,07\% del total de libros en lenguas españolas.

Lenguas extranjeras y/o traducciones. Las traducciones representan el 21,1\% de la producción, destacando el inglés, con 9.673 libros y un $51,0 \%$ de las obras traducidas, en este 2017; seguido del italiano, con 2.085 obras; y el francés, con 1.809.

La producción en lengua extranjera se divide en los siguientes porcentajes: Multilingües, y en inglés, con el 2,03\%, respectivamente; portugués, representado el 0,79\%; francés, el 0,35; italiano, el 0,08\%; alemán, el 0,07\%; y otras lenguas, que en conjunto corresponden al $0,14 \%$ del total editado.

\section{Distinguir quién es quién en la literatura mundial}

Ser reconocido y reconocible. "Una vez elegida y normalizada la firma científica, es recomendable recurrir a una herramienta que permita unificarla”, señala Díaz-Redondo y Frías (2018, p. 252).

De la misma forma que existen identificadores para distinguir un monográfico, como son los ISBN y el ISSN de una publicación periódica, o un recurso en la red —el identificador de recursos uniforme [URI por sus siglas en inglés] — o un objeto digital (el DOI), en años recientes, se ha elevado el desarrollado distintos identificadores digitales para diferenciar a un autor de otro (Díaz-Redondo y Frías, 2018).

Como afirman Lorenzo Escolar y Pastor Ruiz (2012), la identificación universal del nombre de los autores no es un tema nuevo. "Las bibliotecas tienen una larga historia en el uso de los registros de autoridad para identificar sistemáticamente personas y organizaciones, y vincular las diferentes variantes de los nombres dentro de sus catálogos" (Lorenzo y Pastor, 2012, p. 107-108).

Uno de los primeros pasos en la creación de una plataforma de acceso a la información científica es la identificación unívoca de los investigadores. Sólo así es posible asignar las publicaciones - u otros resultados de la actividad científica- a un determinado individuo (Borrego, 2014, p. 3).

Con Internet, el problema de identificar inequívocamente al autor de un documento, se agrava. La información que se alberga, crece de modo exponencial, y seguimos sin controlar un lenguaje documental sencillo que nos permita indexar correctamente el contenido de una información, para —y eso aún no ha cambiado— evitar la generación de ruido en la recuperación de datos, y generar conocimiento en el receptor. 
En la actualidad, coexisten las técnicas, sistemas y procedimientos de identificación de autor por ordenamiento numérico o alfanumérico; modalidades de perfil personal/profesional; así como los recursos mixtos, o acoplados; y los sistemas de identificación de bibliotecas, o el catálogo de autoridades.

\subsection{Sistemas de identificación por codificación numérica o alfanumérica}

Para Lorenzo y Pastor (2012), "por identificador de autor se entiende un código con forma numérica o alfanumérica que se asigna a un autor para identificar de forma inequívoca su producción científica, con independencia de cómo firma o en qué institución trabaja" (p.109).

De ambiente internacional, Número Internacional Normalizado de Nombres (ISNI, por sus siglas en inglés) es una norma ISO 27729:2012, cuyo propósito es identificar individuos y organizaciones establecidos para la creación, producción y distribución de contenidos culturales. ISNI forma parte de una familia de estándares internacionales para la identificación de productos culturales que encierra, entre otros: el Número Internacional Normalizado de Libros (ISBN, por su siglas en inglés); el Número Internacional Normalizado de Publicaciones Seriadas (ISSN, por su siglas en inglés); el Número Internacional Normalizado de Audiovisuales (ISAN, por sus siglas en inglés); y/o el Identificador de Objeto Digital (DOI, por sus siglas en inglés) establecidos los más notorios dígitos de registro de información (Borrego, 2014).

EI ISNI, tiene como objetivo, identificar las identidades públicas de las partes, entendidas como identidades conocidas de las partes incluidas en las industrias de contenidos: medios de creación, producción, gestión, y distribución. Estas partes pueden ser personas naturales, autores institucionales, y/o personas jurídicas o ficticias. "La identidad pública de una persona natural llamada Charles Lutwidge Dodgson, es Lewis Caroll“ (Martín, 2015, p. 10).

Caso español. IraLis, International Registry of Authors-Links to Identify Scientists, es una plataforma apoyada por la Fundación Española para la Ciencia y la Tecnología (FECYT), que se crea con la misión de reunir un registro de autoridades que ayude en la localización de sus diferentes variantes de firma, mejorando la recuperación de información y la visibilidad del perfil profesional de los autores.

IraLIS ha creado una base de datos cuyos registros son generados por los propios autores, rellenando un sencillo formulario a partir del cual se les adjudica un código numérico que permite su identificación (Lorenzo y Pastor, 2012, p. 111). 


\subsection{Sistemas de identificación por perfil personal-profesional}

"El término "perfil de autor" se aplica al conjunto de datos que recogen de forma estandarizada la producción científica o actividad investigadora de un autor: puestos desempeñados, proyectos de investigación, contratos, etcétera" (Lorenzo y Pastor, 2012, p.109).

De ámbito internacional, ResearchID, promovido por Thomson Reuters en 2008. Esta herramienta propone que cada investigador posea un único número identificador, que logre establecer búsquedas bibliográficas sin complicaciones por duplicidad en la identidad del autor.

Researcher ID depende del autor. No hay comprobación absoluta de la autoría de los artículos en dicho sistema de información, ni metodologías para impedir que un autor se registre distintas ocasiones. El autor posee un control general respecto a la información que figura en su perfil público, por lo que "requiere de una mezcla de responsabilidad individual por parte del emisor, y de trabajo crítico por parte del receptor" (Lorenzo y Pastor, 2012, p. 112).

Es decir, a cada perfil profesional/académico se le asigna un número identificador único y a una dirección URL definitiva que facilita la visibilidad y acceso a los trabajos, y a las métricas de cita. Dico identificador se combina de una letra, cuatro dígitos, además del año que se registra.

De acuerdo con Borrego (2014), es necesario:

Hay que destacar que en este proyecto no es el gestor de la base de datos quien asigna un código al autor e intenta agrupar su producción bibliográfica, sino que es el propio investigador quien recupera sus publicaciones de la base de datos en un perfil que crea él mismo y que posteriormente deberá mantener actualizado. Los perfiles de los investigadores son públicos y vienen acompañados de algunos indicadores bibliométricos relativos al número de citas recibidas por cada publicación que sirven como incentivo a los investigadores para la creación de su perfil. (p. 12)

De acuerdo con Borrego (2014) "Google Scholar Citations es una herramienta que permite a los investigadores crear su propio perfil reclamando su producción de entre la indizada por Google Scholar" (p. 14).

En la misma línea Mandiá-Rubal (2015), reconoce qué este sistema

lo integra todo. Crea marca y visibiliza al investigador; le permite la gestión de su perfil profesional; le calcula impactos y visibilidades, actuales y retrospectivas; difunde la producción científica en abierto, a través del motor 
de búsqueda más empleado del Mundo; es independiente de organismos y naciones, su alcance es internacional; permite la cooperación y la colaboración entre los pares; es sencillo de manejar, e interoperable; y además, es gratuito (p. 848).

El Currículum Vítae Normalizado, para el caso español (CVN), se considera una Plantilla estándar, reconocida a nivel nacional, que puntualiza un formato único de exposición de los datos curriculares entre el gremio de investigadores, y que adecúa su funcionalidad con las distintas bases de datos institucionales. Por tanto, al investigador, el CVN, le permite estandarizar su currículo en un formato digital único y común, actualizando de "forma automática su información curricular sin tener que volver a introducirla" - proveyendo el currículum vítae unificado de acuerdo a las convocatorias de financiamiento-; "para sentar las bases de la creación de una red de intereses curriculares comunes para el Sistema Español de Ciencia Tecnología e Innovación" (FECYT, 2018, párr. 1-6).

De acuerdo con Lorenzo y Pastor (2012) el problema de esta plataforma es que

carece de vínculos a bases de datos capaces de proveer el número de citas recibidas, o aportar datos sobre indicadores métricos de las revistas de publicación, aunque esto último se subsana con enlaces a publicaciones registradas, de forma que los autores pueden elaborar indicadores bibliométricos que complementen su evaluación (p. 111).

\subsection{Sistemas de identificación combinados}

Los sistemas de identificación combinados aúnan las dos posibilidades anteriores, respondiendo a la intención de crear sistemas globales de interacción, a la vez que aportan mayor información al usuario sobre el autor que busca.

Aquí las opciones destacadas se incrementan, al tiempo que todas se definen por ser de ámbito internacional. Pudiera parecer que son la tendencia hacia la que caminan los sistemas de identificación de autoridades.

Scopus, el sistema instaurado por la editorial Elsevier en 2006, adopta dos herramientas: Scopus Author Identifier y Author Profile. En contraste del Researcher ID, dicho sistema diferencia substantivamente ambos conceptos, no obstante, se presenten en un mismo folio: "identificador del autor", simbolizado en Scopus Author Identifier; y "perfil del autor", personalizado en Scopus Author Profile. El recurso establece un número identificador exclusivo por cada investigador firmante de artículos indexados en SCOPUS, sin considerar el asentimiento del autor (Lorenzo y Pastor, 2012; Madiá-Rubal, 2015). 
Sobre el mismo tema - de Scopus—, el identificador es establecido por los administradores de la base de datos y, de nuevo, para alcanzar una identificación nominal de cada autor. Aunque se le van asignando las obras automáticamente, el autor puede y debe reclamar su producción para que se le agrupen en una única entrada. "En este caso, el objetivo del identificador es facilitar la búsqueda en la base de datos, y los perfiles no son públicos, sino que sólo están disponibles para los suscriptores" (Borrego, 2014, p. 13).

ORCID, proyecta instituir un reconocimiento abierto, único e independiente, estableciendo identificadores originales enlazables a la productividad científica de cada autor, esto, indistintamente del sistema de información científico en el que aparezca. "ORCID trasciende las disciplinas, las fronteras geográficas nacionales e institucionales, y es el único servicio que trata de asociar su propio identificador con otros identificadores de autor existentes" (Lorenzo y Pastor, 2012, p.113).

Por tanto, se reconoce qué:

La obtención de un identificador ORCID es gratuita y no existe una agencia de registro como sucede con otros identificadores. Cualquier persona puede generar un código ORCID, simplemente registrándose en el sistema y completando una serie de datos (Martín, 2015, p. 11).

El Identificador Estándar Internacional de Nombres (ISNI, por su sigla en inglés), derivado de la familia de identificadores estándar internacionales DOI, Estándar internacional de la numeración audiovisual (ISAN, por su siglas en inglés), ISBN, Código Estándar Internacional de Grabación (ISRC, por sus siglas en inglés), ISSN, ISTC e ISWC, se crea para asegurar un número de identificador único y persistente, que solucione problemas de ambigüedad entre nombres de autoridad. De cara a la catalogación y asignación de las obras, pero también para facilitar la búsqueda de las mismas. Para Borrego (2014), la Agencia de Registro ISNI "ha reservado un conjunto de códigos para el proyecto ORCID de manera que no haya solapamientos en las asignaciones de identificadores. No obstante, a pesar de que un autor tenga un código ISNI no figura necesariamente en la base de datos ORCID" (Borrego, 2014, p. 7).

VIVO, aplicación de origen norteamericano, de código abierto, desarrollada por la Universidad de Cornell en 2003, es una red de perfiles de investigador con más de cien instituciones adheridas, en una docena de países. "VIVO busca mejorar el descubrimiento de autoridades en disciplinas e instituciones" (OCLC, 2018, párr. 27). Conjuntamente, reconoce a las instituciones educativas, al entregar sus datos en acceso abierto, con la finalidad de ser reutilizados, invitando en la mejora de 
aplicaciones colaborativas que facilitan una búsqueda optimizada, nuevos contenidos de cooperación.

Dado que un objetivo clave, tanto de VIVO como de ORCID, es la creación de una infraestructura que haga que la información de los investigadores y sus trabajos académicos estén abiertamente disponibles, ambos están estudiando los beneficios de una colaboración tecnológica más estrecha (Lorenzo y Pastor, 2012, p. 114).

CRIS Current Research Information Systems. Los sistemas de informes y administración de investigación también mantienen un registro sistemático de los nombres de los autores. Existe una superposición funcional de VIVO con los sistemas de perfil de investigador, pero los CRIS generalmente son operados por instituciones y organismos de financiación, y son más comunes en Europa - precisamente por estar bajo el control institucional es difícil estimar el número de investigadores registrados-.

\subsection{El catálogo de autoridades}

"El control de autoridades proporciona consistencia e integridad al catálogo bibliográfico" (Jiménez y García, 2002, p. 25-39), unifica sitios de acceso, formulados de forma distinta en las diferentes fuentes a catalogar; distingue puntos de acceso que se pueden identificar de la misma manera, solventa la homonimia; convierte al catálogo bibliográfico en una red de analogías que admite al usuario conducirse con seguridad a distintas referencias, en la procura de bibliografía de un mismo autor, temática, evento, o institución; dirige al interesado, a través de notas explicativas, a una variedad, condición o grupo de vías, que alcanzan perfeccionar la exploración de información en el catálogo; y deja constancia histórica de las autoridades de un país.

A nivel nacional e internacional, VIAF Virtual International Authority File es la base de datos de autoridades referente.

Se trata de un proyecto colaborativo, de alcance internacional, que enlaza los datos de autoridad proporcionados por las diferentes entidades acreditadas - bibliotecas nacionales y agencias de bibliotecas regionales y transnacionales seleccionadas-, "contribuyendo con su trabajo a nutrir este archivo de autoridad internacional de carácter virtual” (OCLC, 2018, párr. 26).

El sello de garantía que valida la calidad de la información albergada en VIAF es el reconocimiento de las instituciones participantes. 
El objetivo del proyecto es disminuir el coste e incrementar la utilidad de los ficheros de autoridad de las bibliotecas mediante la comparación y la correspondencia entre los ficheros de autoridades de las bibliotecas nacionales (Martín, 2015, p. 8).

VIAF pone la información disponible en Internet. Por tanto,

Se trata de un proyecto alojado por OCLC en el que participan más de veinte bibliotecas nacionales con la finalidad de reducir los costes de esta tarea e incrementar la utilidad de los ficheros de autoridades poniendo en común los mantenidos por diferentes bibliotecas (Borrego, 2014, p. 5).

\section{Conclusiones}

Datos tan simples como la inserción de un segundo apellido unido con guiones, facilitar el año y lugar de nacimiento, la profesión o especialidad, puesto actual, o el enlace a un sistema de identificación como los anteriormente señalados —opción interesante en los casos en los que la inserción de información permitida se circunscribe a espacios muy reducidos, como ocurre en las revistas científicas, y que actualmente subsanan con la inserción obligada del código ORCID—, son posibilidades que aportan ese punto distintivo y contribuyen a crear una marca personal por la que ser reconocidos y reconocibles.

La aparición de los Digital Author Identifiers (DAls) ha supuesto un gran avance para el control de la identificación inequívoca del autor pues bajo un solo código alfanumérico vinculado al investigador es posible controlar no sólo todas las variantes posibles de su firma, sino también toda la producción científica que está indexada bajo ellas. En la actualidad, existen varias opciones, como ORCID, ResearcherID, Scopus Author ID, o IraLIS (Díaz-Redondo y Frías, 2018, 252).

La alfabetización informacional, también es esto. Darse cuenta de las ventajas y oportunidades de Internet, sabiendo operar en este entorno con sus reglas, que necesariamente son distintas a las del mundo analógico.

En Internet, la comunicación en bidireccional, de muchos a muchos; las posibilidades se multiplican; el ámbito de influencia es global; y el crecimiento continúo, además, se debe reconocer, qué "El estudio de las plataformas digitales académicas desde una perspectiva reputacional es más limitado" (Siso-Calvo y Arquero-Avilés, 2020, p. 70). 


\section{Bibliografía}

Acosta-Ortega, L., Montoya-Acosta, L.A., \& Lescay-Arias, M. (2016). Digital identifiers as permanent unique registers for researchers in the university context. Santiago: revista electrónica, vol. 141, pp. 596-609.

Barroso-Osuna, J., Llorente-Cejudo, C. y Palacios-Rodríguez, A. (2020). Competencia e identidad digital. Percepciones del profesorado en formación. AULA, Revista de Humanidades y Ciencias Sociales, 66 (2), 53-64

Borrego, Á. (2014). Sistemas de identificación unívoca de investigadores: traducción del informe realizado en mayo de 2013 por encargo del Consorci de Biblioteques Universitàries de Catalunya (CBUC). En: Manuales y guías, repositorios. [Barcelona]: CRUE Universidades Españolas; Red de Bibliotecas REBIUN.

Díaz-Redondo, C., y Frías Montoya, J.A. (2018). Criterios para la elección, normalización y unificación de la firma científica. Revista ORL, vol. 9, núm. 4, pp. 251-256.

ESPAÑA, Biblioteca Nacional de España (2018). Catálogo BNE, búsqueda de autoridades. Recuperado de http://catalogo.bne.es/uhtbin/authoritybrowse.cgi

ESPAÑA, Ministerio de Cultura y Deporte (2018). Panorámica de la Edición Española. Recuperado de https://bit.ly/3uN3hp4

ESPAÑA, Ministerio de Educación y Cultura (1999). Reglas de catalogación. Madrid: Boletín Oficial del Estado.

FECYT, Fundación Española para la Ciencia y la Tecnología (2018). CVN Currículum Vítae Normalizado. Recuperado de https://cvn.fecyt.es/presentacion/que-es-cvn

García, C. (2014). Els identificadors a la literatura científica. Barcelona: Universitat Politècnica de Catalunya, Servei de Biblioteques, Publicacions i Arxius.

García-Peñalvo, F. J. (2019). Las dimensiones de la identidad digital de un investigador. III Jornadas Investigación e Innovación Educativa (12 de diciembre de 2019, Albacete, España). Salamanca, España: Grupo GRIAL. https://bit.ly/34cljIO. doi:10.5281/zenodo.3570884

Genovés, P. (2017). Perfiles de autor en repositorios institucionales $=$ Author profiles in institutional repositorie. Palabra clave, vol. 7, núm. 1, pp. 1-14.

INE, Instituto Nacional de Estadística (España). INEbase [en línea]. [Madrid]: INE. Recuperado de https://bit.ly/3mPopbB 
Jiménez Pelayo, J., \& García Blanco, R (2002). El catálogo de autoridades: creación y gestión en unidades documentales. Gijón, Asturias: Trea.

Lorenzo-Escolar, N., \& Pastor-Ruiz, F (2012). Un análisis de los principales sistemas de identificación y perfil para el personal investigador. Aula Abierta, vol. 40, núm. 2, pp. 107-118.

Mandiá-Rubal, S. (2015). Nuevos activos en la comunicación de organizaciones científicotecnológicas: perfiles personales en redes profesionales. La pantalla insomne. [Tenerife]: Universidad de La Laguna.

Martín, S.G. (2015). Sistemas digitales de identificación de autores/investigadores. V Encuentro Nacional de Catalogadores Desafíos Actuales de los Servicios Técnicos en las Bibliotecas Argentinas 7, 8 y 9 de octubre de 2015.

Mistral, F., \& Mauger-Perez, I. (2018). Autorités Identifiants personnes \& structures. Agence Bibliographique de l'Enseignement Supérieur, pp. 1-25.

OCLC Community Center (2018). Registering Researchers in Authority Files. Recuperado de https://www.oclc.org/research/themes/research-collections/registering-researchers.html

Picco, P., \& Ortiz Repiso, V. (2012). RDA, el nuevo código de catalogación: cambios y desafíos para su aplicación. Revista Española De Documentación Científica, 35(1), 145-173. https://doi.org/10.3989/redc.2012.1.848

Portugal, R., y Aguaded, I. (2020). Competencias mediáticas y digitales, frente a la desinformación e infoxicación. Razón Y Palabra, 24(108). https://doi.org/10.26807/rp.v24i108.1658

Poveda, A., Rasero, V., Fernández del Pino, B., \& Martín, J. L. (2017). Sincronización en el control de Autoridades Repositorio/ CRIS. https://gredos.usal.es/bitstream/handle/10366/135611/ECA PovedaA Sincronizaci\%C3\%B3n.pdf ?sequence $=1$

Siso-Calvo, B., y Arquero Avilés, R. (2020). Plataformas digitales y reputación académica. Ibersid: Revista De Sistemas De Información Y Documentación 14(1), 69-77. Recuperado a partir de https://ojs.ibersid.eu/index.php/ibersid/article/view/4692

Sandberg, J., \& Jin, Q. (2016). How Should Catalogers Provide Authority Control for Journal Article Authors? Name Identifiers in the Linked Data World. Cataloging and Classification Quarterly, 54(8), pp. 537-552.

UCLA Library (2018). Research Visibility: how to raise the visiblity of your research and establish your name in an academic field. Recuperado de http://guides.library.ucla.edu/seo/profiles 